\title{
openheart Ultrasound-guided antecubital vein approach for right heart catheterisation in a Brazilian tertiary centre
}

\author{
Felipe Homem Valle (D) ,1,2 Rodrigo Vugman Wainstein,, ${ }^{3,4}$ Bruno Silva Matte, ${ }^{3}$ \\ Sandro Cadaval Gonçalves, ${ }^{3}$ Luiz Carlos C Bergoli, ${ }^{3}$ Ana Maria Rocha Krepsky, ${ }^{3}$ \\ Fernando Pivatto Junior, ${ }^{4}$ Gustavo Neves de Araujo, ${ }^{3,4}$ \\ Guilherme Pinheiro Machado (D) , ${ }^{4}$ Marco Vugman Wainstein ${ }^{3,4}$
}

To cite: Valle FH, Wainstein RV, Matte BS, et al. Ultrasoundguided antecubital vein approach for right heart catheterisation in a Brazilian tertiary centre. Open Heart 2020;7:e001181. doi:10.1136/ openhrt-2019-001181

Received 5 October 2019 Revised 2 January 2020 Accepted 5 January 2020

Check for updates

\section{Author(s) (or their} employer(s)) 2020. Re-use permitted under CC BY-NC. No commercial re-use. See rights and permissions. Published by BMJ.

${ }^{1}$ Cardiology, St. Michael's Hospital, Toronto, Ontario, Canada

${ }^{2}$ Cardiology, Mount Sinai Hospital/University Health Network, Toronto, Ontario, Canada

${ }^{3}$ Cardiology, Hospital de Clinicas de Porto Alegre, Porto Alegre, Brazil

${ }^{4}$ Postgraduate Program in Cardiology and Cardiovascular Sciences, UFRGS, Porto Alegre Brazil

\section{Correspondence to}

Dr Felipe Homem Valle; felipe. valle@mail.utoronto.ca

\section{ABSTRACT}

Objective As a parallel to the radial approach for left heart catheterisation, forearm veins may be considered for the performance of right heart catheterisation. However, data regarding the application of this technique under ultrasound guidance are scarce. The current study aims to demonstrate the feasibility of right heart catheterisation through ultrasound-guided antecubital venous approach in the highly heterogeneous population usually referred for right heart catheterisation.

Methods Data from consecutive right heart catheterisations performed at an academic centre in Brazil, between January 2016 and March 2017 were prospectively collected.

Results Among 152 performed right heart catheterisations, ultrasound-guided antecubital venous approach was attempted in 127 (84\%) cases and it was made feasible in $92.1 \%$ of those. Yet, there was no immediate vascular complication with the antecubital venous approach in this prospective series.

Conclusions Ultrasound-guided antecubital venous approach for the performance of right heart catheterisation was feasible in the vast majority of cases in our study, without occurrence of vascular complications.

\section{INTRODUCTION}

Since Werner Forssman performed the first human cardiac catheterisation accessing his own right heart through a left antecubital vein, in $1929,{ }^{1}$ the field has evolved tremendously and right heart catheterisation has become an important and frequently performed procedure in a wide range of medical conditions. ${ }^{2}$ Currently, the femoral or internal jugular veins are the most frequently accessed sites used for the performance of right heart catheterisations and, ${ }^{2}$ as a parallel to radial approach for left heart catheterisation, ${ }^{3-5}$ forearm veins have recently become a new potential route for the performance of right heart catheterisations. ${ }^{6}$

The data supporting the utilisation of the antecubital venous approach for right heart

\section{Key questions}

What is already known about this subject?

- Right heart catheterisations are mostly performed from central venous access sites. Utilisation of peripheral arm access, such as the antecubital veins, in order to perform right heart catheterisation minimises the invasiveness of the procedure. Ultrasound guidance to obtain antecubital venous access might further expand the applicability of the intervention.

What does this study add?

- In a consecutive series of more than 100 patients, our study demonstrated high success rate in performing right heart catheterisation from the ultrasound-guided antecubital venous approach.

How might this impact on clinical practice?

- Our study results encourage the adoption of ultrasound-guided antecubital venous approach as an alternative access route for the performance of right heart catheterisation.

catheterisation are mainly derived from a series in which eligibility for such approach was based on visualisation/palpation of the antecubital veins. ${ }^{6-15}$ Hence, the applicability of the antecubital venous approach for the highly heterogeneous populations usually referred for right heart catheterisation is unclear. Further, the use of ultrasound guidance for venous access acquisition is currently strongly encouraged, ${ }^{16}$ potentially reducing the incidence of local vascular complications and improving the success rates of venous punctures. Therefore, we hypothesised that the advantages of ultrasound guidance utilisation could potentially be translated for the performance of right heart catheterisations from the antecubital venous approach, expanding the applicability of this technique to a broader range of patients. The purpose of this study is to report our experience in ultrasound-guided antecubital venous access right heart catheterisations. 


\section{METHODS}

In January 2016, a programme of antecubital venous approach for right heart catheterisation began in our institution and it became our default venous access for right heart catheterisation. When simultaneous left heart catheterisation was necessary, preference was given for combined antecubital venous and radial artery approaches. Notably, patients with upper arm haemodialysis arteriovenous fistulae were not considered eligible for the antecubital venous approach. In our institution, endomyocardial biopsies and electrophysiological studies are performed exclusively through jugular and femoral veins, respectively; thus, when any of those procedures was made necessary, in addition to right heart catheterisation, patients were considered ineligible for the antecubital venous approach.

Our study aimed to address the proportion of patients in which the antecubital venous approach was undertaken and to report the reasons for deferring the antecubital venous approach for right heart catheterisation. Further, we aimed to assess the rate of completion of right heart catheterisation from an antecubital vein and to assess the success rate in obtaining venous access through the antecubital venous approach, which was ascertained as successful insertion of a 7-French sheath in an antecubital vein. Data regarding demographic and procedures characteristics were prospectively collected.

\section{Study protocol}

Patients who were referred for right heart catheterisation came to the catheterisation laboratory after 8 hours of fasting. After tourniquet application to the upper arm, the right antecubital fossa veins were assessed with a portable ultrasound (FUJIFILM SonoSite M-Turbo, WA, USA). A cross-sectional view of the brachial artery was taken as landmark, followed by identification of both cephalic and basilic veins. Preference was given to the basilic vein rather than the cephalic vein since the former is usually the larger antecubital vein and the latter usually enters the axillary vein in a sharp angle that may be unfavourable for catheter advancement. Under local anaesthesia and guided by ultrasound visualisation, the selected vein was punctured with a 21-gauge needle (Micropuncture, Cook, IN, USA), a 0.018-inch wire was then advanced through the vessel and intraluminal wire positioning was confirmed with longitudinal ultrasound assessment of the instrumented vessel (figure 1). Then, a $10 \mathrm{~cm}$, 5-French sheath was inserted and this was further exchanged to a $10 \mathrm{~cm}, 7$-French sheath through a 0.035 -inch wire. Under fluoroscopy, a 7-French Swan-Ganz (Edwards, CA, USA) catheter was advanced to the pulmonary artery and haemodynamic evaluations were performed. After catheter withdrawal, the sheath was removed; manual compression was performed for $5 \mathrm{~min}$ at the puncture site and the site was dressed. Patients were discharged 10-20 min after the procedure, unless left heart catheterisation had also been performed. In that case, the local protocol for radial artery dressing was followed.

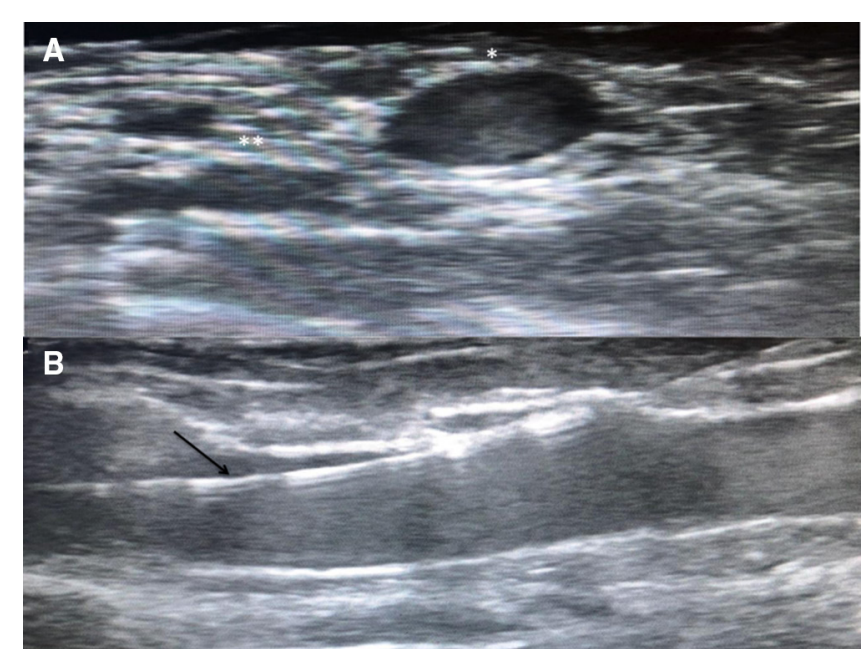

Figure 1 Ultrasound-guided antecubital venous access. A: Identification of vascular landmarks at cross-sectional view of the antecubital fossa: basilic vein (asterix) and brachial artery (double-asterisks); B: Confirmation of intravascular wire positioning (arrow).

\section{Statistical analysis}

Continuous variables were expressed as mean values $\pm S D$, after normality of data was assessed with the Shapiro-Wilk normality test. Categorical variables were expressed as numbers of patients and percentages. SPSS V.18.0 was used for statistical analysis.

\section{RESULTS}

Between January 2016 and March 2017, 152 right heart catheterisations were consecutively performed. Twenty-five $(16 \%)$ patients were considered ineligible for the antecubital venous approach due to the following reasons: haemodialysis arteriovenous fistula $(n=11)$; need for prolonged invasive monitoring with consequent prolonged maintenance of the pulmonary artery catheter in place $(n=4)$; simultaneous electrophysiological study $(\mathrm{n}=2)$; simultaneous endomyocardial biopsy ( $\mathrm{n}=2)$; simultaneous pulmonary artery angioplasty $(n=1)$; previous axillary lymphadenectomy $(n=1)$; others $(n=2)$. In the remaining 127 cases, under ultrasound guidance, the antecubital venous approach was attempted and the demographic characteristics as well as right heart catheterisation clinical indications are described in table 1.

Among the 127 cases in which the antecubital venous access was attempted, successful insertion of a 7-French sheath in an antecubital vein was achieved in 122 (96\%) patients. In the five (4\%) cases in which failure to obtain antecubital venous access occurred, four were related to venous puncture failure and the remaining failure was related to inadvertent brachial artery puncture: in these five cases, after 3-5 min of manual compression, right heart catheterisations were performed from another venous access site, according to operator's preference. Within the 122 cases in which antecubital venous access was obtained, successful performance of right heart catheterisation from the antecubital venous approach was feasible in 117 
Table 1 Demographic characteristics and clinical reasons for right heart catheterisation

\begin{tabular}{ll}
\hline Age (years) & $\mathbf{5 5 \pm 1 4}$ \\
\hline Female gender (\%) & $73(57)$ \\
\hline Weight $(\mathrm{kg})$ & $73 \pm 18$ \\
\hline Height $(\mathrm{m})$ & $1.63 \pm 0.1$ \\
\hline Body mass index (kg/m²) & $26.8 \pm 5.8$ \\
\hline Pulmonary hypertension assessment (\%) & $51(40)$ \\
\hline Heart failure assessment (\%) & $25(20)$ \\
Lung transplantation work-up (\%) & $19(15)$ \\
\hline Heart transplantation work-up (\%) & $15(12)$ \\
\hline Congenital heart disease assessment (\%) & $17(13)$ \\
\hline
\end{tabular}

(92.1\%) patients (figure 2). The reasons for unsuccessful performance of right heart catheterisation from the antecubital venous approach were related to subclavian vein thrombosis $(n=3)$, axillary vein thrombosis $(n=1)$ and superior vena cava thrombosis $(n=1)$. The basilic, cephalic and brachial veins were used in $110(94 \%), 6(5 \%)$ and $1(1 \%)$ cases, respectively. Simultaneous left heart catheterisation with or without coronary angiography was performed in 57 $(45 \%)$ cases and the radial artery approach was the arterial access route in all those cases. In our cohort, there was no immediate significant vascular complication, such as significant bleeding, significant haematoma, limb ischaemia or needing for prolonged stay at the catheterisation laboratory, after attempted antecubital venous approach right heart catheterisation.

\section{DISCUSSION}

In the present study, we prospectively examined the feasibility of right heart catheterisation from ultrasoundguided antecubital venous approach in a public hospital in Brazil. Overall, in our cohort, the antecubital venous approach was undertaken in $84 \%$ of right heart catheterisations performed in our institution and it was made possible in $92 \%$ of these cases. We also examined the rate of success in obtaining antecubital venous access under ultrasound guidance, which was $96 \%$ in our study. Further, we examined the reasons for antecubital

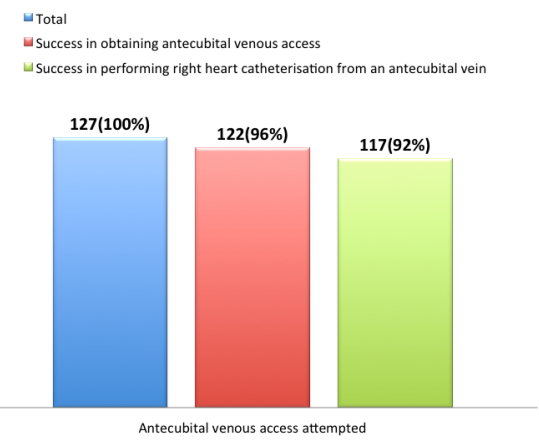

Figure 2 : Rates of success in obtaining antecubital venous access under ultrasound guidance ( $(\mathrm{ed})$ and in performing right heart catheterisation from an antecubital vein (green). venous approach failure. Despite adequate cannulation of an antecubital vein, right heart catheterisation was not completed from the antecubital venous approach in five cases due to unforeseen proximal vessel occlusions.

In our study, which included 127 attempts to perform right heart catheterisation from antecubital venous approach, obtained under ultrasound guidance, absence of vascular complications was observed. Although right heart catheterisation is considered a low-risk procedure with low incidence of adverse events, local vascular complications are responsible for the majority of those events and there are established site-specific risks. ${ }^{2}$ Further, previous small studies demonstrated reduced incidence of vascular complications, early ambulation and greater comfort to patients with the antecubital venous approach for right heart catheterisation. ${ }^{6-13}$

Combined left and right heart catheterisations were performed in $45 \%$ of the cases in our study and the radial artery approach was the arterial route for left heart catheterisation in all those cases. Preference for the radial artery approach for left heart catheterisation, particularly for percutaneous coronary interventions, is currently strongly encouraged. ${ }^{1516}$ In this setting, the opportunity of performing combined right and left heart catheterisations with limited manipulation to the arm and forearm might further minimise the invasiveness of such interventions, with potential greater comfort to patients.

In our cohort, attempts to access antecubital veins were successful in $96 \%$ of the cases and the five observed failures occurred within the first 50 attempts to perform right heart catheterisation from antecubital veins in our institution, suggesting that our unsuccessful attempts might, in fact, represent our learning curve in obtaining antecubital venous access under ultrasound guidance. Similarly, Harwani et al reported $100 \%$ success rate with ultrasound-guided peripheral venous access strategy for both right heart catheterisations and endomyocardial biopsies. Notably, our protocol, as well as Harwani et $a l$ s, ${ }^{8}$ which similarly to ours used ultrasound guidance to obtain antecubital venous access, provides information on the adoption of antecubital venous approach as the preferred route for right heart catheterisations. The previous series of right heart catheterisations from the 'forearm approach' included either individuals with palpable/visible antecubital veins or individuals in which forearm venous access had been obtained before the procedure. ${ }^{69-14}$ Indeed, in the only study that clearly reported the overall success rate of the antecubital approach, a $15 \%$ access crossover rate was observed. ${ }^{6}$ Hence, the nature of these previous series preclude definitive conclusion of the feasibility of the antecubital venous approach in the highly heterogeneous population of patients that may require right heart catheterisation.

The present study has limitations. This is a singlecentre case series and despite prospective data collection, this is an observational study without a control group for comparison. Further, although our study reports data on consecutive patients, we used a convenience sample. 
Therefore, our findings need to be interpreted in an exploratory fashion and further studies should address the reproducibility of our results.

In conclusion, in our prospective series, performance of right heart catheterisations from antecubital venous access, obtained under ultrasound guidance, was feasible in the vast majority of cases. Moreover, the absence of significant vascular complications observed in our study encourages further studies in this field.

Contributors FHV, RVW and MVW planned the study. FHV, RVW, BSM and MVW conducted the study procedures. FHV, FPJ and GNdA performed the statistical analysis. FHV wrote the manuscript draft, which has been approved by all the authors.

Funding The authors have not declared a specific grant for this research from any funding agency in the public, commercial or not-for-profit sectors.

Competing interests None declared.

Patient consent for publication Not required.

Ethics approval The local institutional Research Ethics Board has approved the study protocol.

Provenance and peer review Not commissioned; externally peer reviewed.

Data availability statement Data obtained for this study are available on reasonable request.

Open access This is an open access article distributed in accordance with the Creative Commons Attribution Non Commercial (CC BY-NC 4.0) license, which permits others to distribute, remix, adapt, build upon this work non-commercially, and license their derivative works on different terms, provided the original work is properly cited, appropriate credit is given, any changes made indicated, and the use is non-commercial. See: http://creativecommons.org/licenses/by-nc/4.0/.

Correction notice This article has been updated since first published to correct a spelling error in the title.

\section{ORCID iDs}

Felipe Homem Valle http://orcid.org/0000-0001-7102-6644

Guilherme Pinheiro Machado http://orcid.org/0000-0002-5514-2562

\section{REFERENCES}

1 Forssmann W. Die Sondierung des Rechten Herzens. Klin Wochenschr 1929;8:2085-7.

2 Ranu H, Smith K, Nimako K, et al. A retrospective review to evaluate the safety of right heart catheterization via the internal jugular vein in the assessment of pulmonary hypertension. Clin Cardiol 2010;33:303-6.

3 Mehta SR, Jolly SS, Cairns J, et al. Effects of radial versus femoral artery access in patients with acute coronary syndromes with or without ST-segment elevation. J Am Coll Cardiol 2012;60:2490-9.

4 Romagnoli E, Biondi-Zoccai G, Sciahbasi A, et al. Radial versus femoral randomized investigation in ST-segment elevation acute coronary syndrome: the RIFLE-STEACS (Radial Versus Femoral Randomized Investigation in ST-Elevation Acute Coronary Syndrome) study. J Am Coll Cardiol 2012;60:2481-9.

5 Valgimigli M, Gagnor A, Calabró P, et al. Radial versus femoral access in patients with acute coronary syndromes undergoing invasive management: a randomised multicentre trial. Lancet 2015;385:2465-76.

6 Roule V, Ailem S, Legallois D, et al. Antecubital vs femoral venous access for right heart catheterization: benefits of a flashback. Can J Cardiol 2015 ;;31:1497.e1-1497.e6. Dec 1.

7 Waheed O, Sharma A, Singh M, et al. Antecubital fossa venous access for right heart catheterization. J Invasive Cardiol 2017;29:169-74.

8 Harwani N, Chukwu E, Alvarez M, et al. Comparison of brachial vein versus internal jugular vein approach for access to the right side of the heart with or without myocardial biopsy. Am J Cardiol 2015:116:740-3.

9 Lee SH, Chun KJ, Lee DS, et al. Right cardiac catheterization using the antecubital fossa vein in Korean patients. Korean Circ $J$ 2016;46:207-12.

10 Tebet MA, Andrade PBde, Andrade MVAde, de APB, de AMVA, et al. Segurança e eficácia da cateterização cardíaca direita e esquerda pelo acesso transradial comparado ao transfemoral: experiência inicial. Rev. Bras. Cardiol. Invasiva 2008:16:317-21.

11 Lo TSN, Buch AN, Hall IR, Hildick-Smith DJ, et al. Percutaneous left and right heart catheterization in fully anticoagulated patients utilizing the radial artery and forearm vein: a two-center experience. J Interv Cardiol 2006;19:258-63.

12 Williams PD, Palmer S, Judkins C, et al. Right and left heart catheterization via an antecubital fossa vein and the radial artery-a prospective study. J Invasive Cardiol 2014;26:669-73.

13 Gilchrist IC, Kharabsheh S, Nickolaus MJ, et al. Radial approach to right heart catheterization: early experience with a promising technique. Catheter Cardiovasc Interv 2002;55:20-2.

14 Gilchrist IC, Moyer CD, Gascho JA. Transradial right and left heart catheterizations: a comparison to traditional femoral approach. Catheter Cardiovasc Interv 2006;67:585-8.

15 Neumann F-J, Sousa-Uva M, Ahlsson A, et al. 2018 ESC/ EACTS guidelines on myocardial revascularization. Eur Heart $J$ 2019;40:87-165.

16 Mason PJ, Shah B, Tamis-Holland JE, et al. An update on radial artery access and best practices for transradial coronary angiography and intervention in acute coronary syndrome: a scientific statement from the American Heart Association. Circ Cardiovasc Interv 2018.;;11:e000035. Sep. 